Rabaska

Revue d'ethnologie de l'Amérique française

\title{
« La Belle qui fait la morte ». Emprunt et adaptation : les versions en langue bretonne
}

\section{Donatien Laurent}

Numéro 1, 2003

URI : https://id.erudit.org/iderudit/201602ar

DOI : https://doi.org/10.7202/201602ar

Aller au sommaire du numéro

Éditeur(s)

Société québécoise d'ethnologie

ISSN

1703-7433 (imprimé)

1916-7350 (numérique)

Découvrir la revue

Citer cet article

Laurent, D. (2003). « La Belle qui fait la morte ». Emprunt et adaptation : les versions en langue bretonne. Rabaska, (1), 21-29.

https://doi.org/10.7202/201602ar d'utilisation que vous pouvez consulter en ligne. 


\section{«La Belle qui fait la morte ». Emprunt et adaptation : les versions en langue bretonne}

DONATIEN LAURENT

Université de Bretagne occidentale, Brest

La situation de la culture populaire en Basse-Bretagne ${ }^{1}$ rappelle, à certains égards, celle du Canada français. Deux communautés linguistiques s'y côtoient, l'une française, l'autre bretonne. Le français, langue unique de l'administration et de l'enseignement, domine largement la vie publique ; l'oral et l'écrit s'y équilibrent et se complètent. Le breton, langue essentiellement orale, langue du peuple des campagnes, plus concrète et plus imagée, survit pourtant à tous les assauts, dans le domaine de l'oralité quotidienne.

Les deux langues ont été riches d'une ancienne culture, et en particulier d'une tradition orale chantée qui leur était propre. Les emprunts de l'une à l'autre, dans ce domaine spécifique, se sont faits presque exclusivement du breton au français ; mais il n'y a emprunt, dans cette société où domine l'oral, qu'accompagné d'une recomposition et d'une élaboration nouvelle qui en modifient souvent profondément le contenu ${ }^{2}$. Encore faut-il noter que, dans le domaine de la chanson, ces emprunts se sont limités d'eux-mêmes, car les deux traditions ont des caractères littéraires bien différents. En breton, la poésie orale chantée à caractère narratif est avant tout soucieuse de vérité, de témoignage authentique plutôt que de fiction. Elle est en quête de renseignements sur les héros et les lieux de l'action : elle veut les noms, les prénoms, les lieux précis, les situations sociales, tout ce qui garantit la réalité vécue et l'authenticité de la situation évoquée. La chanson française, au contraire, a tendance à typifier personnages et lieux. C'est la vérité psychologique, humaine qui l'intéresse, le drame social qui touche tout individu sans distinction d'origine... Il sera donc intéressant de suivre le passage d'une

1. On appelle Basse-Bretagne, par opposition à la Haute-Bretagne ou pays gallo, la partie occidentale de la péninsule armoricaine, à l'ouest d'une ligne Saint-Brieux-Vannes, où l'on parle une langue celtique, le breton.

2. Cf. J.M.Guilcher, "Conservation et renouvellement dans la culture paysanne ancienne de BasseBretagne», Arts et Traditions populaires, 1967, $\mathrm{n}^{\circ} 1$, pp.1-18, et surtout, du même, Rondes, branles, caroles. Le Chant dans la danse, Brest, CRBC et ADP, 2003, à paraître. 
forme à l'autre lorsqu'il y a emprunt. Quels thèmes narratifs privilégie-ton ? Quels traitements leur fait-on subir?

L'exemple que je voudrais présenter, et qui s'est imposé à moi dans le contexte de cette rencontre, est celui d'une chanson française traditionnelle - «La Belle qui fait la morte pour son honneur garder» - dont la descendance est riche dans les trois traditions narratives chantées qui nous intéressent ici : française ancienne, d'où elle provient; bretonne celtique, où elle est passée par traduction et adaptation, tant du texte que de la mélodie ; française d'Amérique enfin, où elle a fait également une brillante carrière.

J'en rappelle l'argument :

Une jeune fille, qui se promène dans son jardin, est courtisée par trois capitaines. Le plus jeune la fait monter sur son cheval et l'amène à Paris dans une hôtellerie. Il lui fait servir à manger et à boire et lui annonce qu'elle passera la nuit avec lui et les deux autres capitaines. Au milieu du souper, elle tombe morte. Les trois comparses décident de l'enterrer au jardin de son père. Mais, trois jours plus tard, son père qui la recherche dans son jardin l'entend qui l'appelle :

- Ouvrez, ouvrez ma tombe, pour mon honneur garder

Georges Doncieux qui en a fait l'étude critique en 1893 dans la revue Mélusine (vol. 6, col. 145-151, 220-224) puis, plus complètement, en 1904 dans son Romancero populaire de la France (pp. 269-279), voyait dans cette chanson « la plus poétique qui soit peut-être dans la poésie populaire de la France » (cf. p. 276). Au moins fut-elle l'une des plus prisées si l'on en juge par le nombre des versions que les collectes folkloriques des $\mathrm{XIX}^{\mathrm{e}}$ et $\mathrm{XX}^{\mathrm{e}}$ siècles nous en ont fait connaître. Patrice Coirault en recense quelques 150 versions, dont une dizaine recueillie en Bretagne gallo ${ }^{3}$. De son côté, Conrad Laforte en a identifié plus de 120 textes, originaires de toute 1'Amérique francophone ${ }^{4}$. Soit environ 270 à 300 versions françaises, étroitement solidaires et stables, tant du point de vue textuel que mélodique.

Autre indice d'une longue popularité : le fait qu'elle soit passée dans les langues et dialectes romans en contact direct avec le français (occitan, catalan, franco-provençal), franchissant même les Alpes et les Pyrénées puisqu'on en trouve des versions jusqu'en Piémont italien et au pays basque. De la même façon, en Bretagne, les nombreuses versions françaises voisinent le long de la frontière linguistique avec des versions en langue bretonne directement adaptées des premières dont elles ont même conservé la mélodie

3. Patrice Coirault, Répertoire des chansons françaises de tradition orale, $\mathrm{I}, \mathrm{n}^{\circ} 1307$.

4. Conrad Laforte, Le Catalogue de la chanson folklorique française, II, A25. 
ou des éléments de celle-ci ${ }^{5}$. Ce sont ces versions bretonnes que je vais maintenant évoquer.

J'ai pu en réunir une quinzaine. Elles s'organisent en deux séries bien distinctes provenant de deux terroirs de dialecte différent, le Trégor et le pays vannetais, sur les deux côtés nord et sud de la péninsule armoricaine. À l'exception de six d'entre elles, recueillies au cours du dernier demi-siècle, les neuf autres appartiennent à des collectes anciennes (1850-1915), restées pour l'essentiel manuscrites.

Avant d'en venir à l'examen de ces deux séries en langue bretonne, faisons un point rapide sur les versions françaises qui ont cours en Morbihan bretonnant, région de transition où les chanteurs connaissent et pratiquent les deux répertoires français et breton. Voici, par exemple, une version de la Chapelle-Neuve, près de Baud, que j'ai recueillie à la fin des années 1950 . On la distingue difficilement de ses sœurs de la France de l'Ouest ou d'Amérique francophone : même intrigue, même formule strophique, même dessin mélodique ${ }^{6}$ que je découpe en six éléments - ab/cd/be correspondant aux six hémistiches de chaque couplet pour mieux en suivre l'évolution.

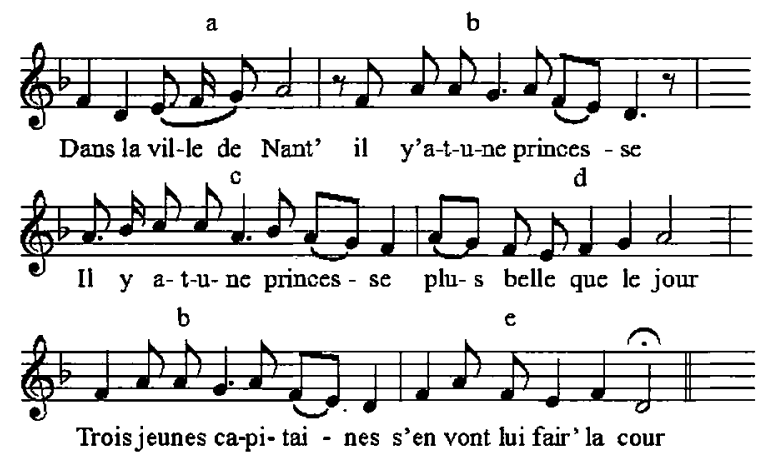

5. Dans sa thèse récente sur la contribution bretonne à l'enquête Fortoul de 1852 concernant les Poésies populaires de la France, Didier Bécam en recense 25 versions françaises et 10 en langue bretonne (cf. D. Bẻcam, « Enquête officielle sur les poésies populaires de la France (1852-1876) : collectes bretonnes de langue française », janvier 2000, vol. 1, pp. 181-185, « La Belle qui fait la morte »).

6. C'est bien la même mélodie-type que l'on retrouve associée à la plupart des versions françaises de «La Belle qui fait la morte », des Alpes au Nivernais, de Rennes à Vendôme et à la Vendée... C'est aussi celle de la belle version de Louisiane que Jean-Michel Guilcher $m$ 'a fait connaitre dans les collections de l'Institut de phonétique de Paris lorsque je commençai cette recherche. Je l'ai retrouvée chez M. et $R$. d'Harcourt (Gaspé, Beauce) et chez M. Barbeau (Éboulements, Bas-Saint-Laurent). Robert Bouthillier et Vivian Labrie, à qui je suis redevable de plusieurs versions canadiennes-françaises, l'ont également rencontrée à plusieurs reprises dans leurs enquêtes. C'est vraisemblablement cette mélodie que Patrice Coirault désigne par «l'air habituel » (P. Coirault, Répertoire, I, 1307, note a, p. 151). 
« Dans la ville de Nantes », collection Donatien Laurent, Marie Le Gal, La Chapelle-Neuve (Morbihan), 1956.

La structure est simple, tant du point de vue textuel que mélodique : un tercet de vers longs (douze pieds) avec césure au milieu $(6+6)$. Doncieux les présente ainsi : " le premier, féminin (donc à césure masculine), qui ne rime pas; le deuxième et le troisième masculins (par conséquent à césure féminine), rimant ensemble » (Mélusine 6, 1893, col. 146).

Soit la formule strophique suivante

MF $6+6$

FM $6+6$

FM $6+6$

Les versions bretonnes vont suivre de très près le texte français, gardant à l'occasion certains passages tels quels, comme l'invitation à monter sur son cheval que fait le capitaine à la belle (Montez, montez la belle dessus mon cheval gris) qui est conservée en français dans les deux séries, trégorroise et vannetaise. C'est par contre du côté de la mélodie que l'invention s'est le plus exercée : elle va jouer librement des différentes séquences mélodiques correspondant aux six hémistiches, les combinant différemment pour mieux s'adapter aux modèles proposés par la tradition locale.

\section{A. Les versions vannetaises}

Ce sont les plus nombreuses et les plus proches du modèle français. Ce sont aussi les mieux connues car deux versions, en provenance respectivement de Belz et de Languidic, en ont été publiées, la première dès 1893 dans la revue Mélusine, vol. 6, col. 181-2: Er plah é hoarn hé inour (La Fille qui garde son honneur), l'autre en 1912 dans Les Chansons de France, tome 6, pp. 556-557 : Barh é Chateaubriant (À Chateaubriant), version reprise l'année suivante par Loeiz Herrieu dans son recueil de Chansons populaires $d u$ pays de Vannes, $2^{\mathrm{e}}$ série, pp. 84-85. À la même époque, Yves Le Diberder en a, de son côté, transcrit trois versions à Pont-Scorff, Landévant et Plouay, avec leurs mélodies. Une sixième version (texte seul) est notée à Plouhinec par l'abbé J. -L. Larboulette, toujours dans ces mêmes années du début du $\mathrm{XX}^{\mathrm{e}}$ siècle. Quatre autres versions vannetaises ont été notées, l'une à Etel près d'Auray au milieu des années 1950 (v. ci-dessous), une autre à Brech en 1970 et deux à Bubry en 1976 ${ }^{7}$. Voici, pour illustrer cette première série, la version d'Etel. On y retrouve les trois vers longs à forte césure des versions françaises, mais les segments mélodiques sont traités différemment.

7. La première par Paul Le Goff, la seconde par moi-même, les deux autres par Jean-Yves Monnat. 


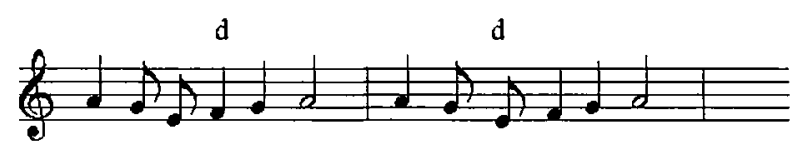

Nag ur verhig ye- vank, merh d'ur priñs a Ba - ris,
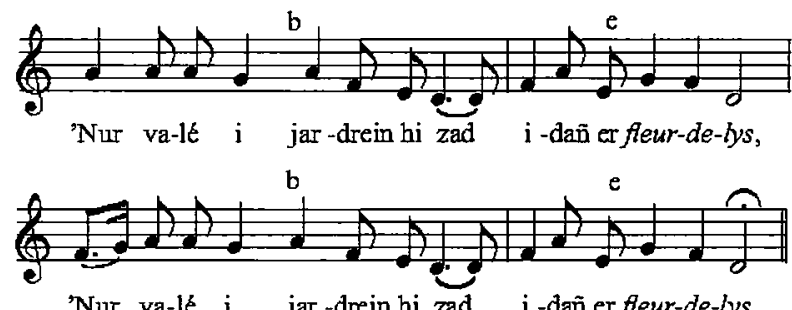

'Nur va-lé i jar-drein hi zad i -dañ er feur-de-lys.

\section{« Nag ur verhig yevank », collection Paul Le Goff, Etel (Morbihan), 1955.}

1 Nag ur verhig yevank, merh d'ur priñs a Baris, 'n ur valé i jardrein hi zad idañ er fleur-de-lys; (bis)

'n ur valé i jardrein hi zad idañ er fleur-de-lys, trei mab d'ur habiten yevank e ri er lis dehi. (bis)

5 Trei mab d'ur habiten yevank e ri er lis dehi hag er yevankekan anè lavaras dei un dé : (bis)

Har er yevankekan anè lavaras dei un dé :

- Montez, montez, montez, la belle, mont à mon cheval gris/(bis)

Montez, montez, montez, la belle, mont' à mon cheval gris,

10 ha mé hou kasei mi du-hont d'er gér vras a Baris! (bis) et je vous amènerai là-bas dans la grande ville de Paris!

Er gér vras a Baris a pe oent alrriùet,

Barh in un ostalerei vras ou deoé dichennet. (bis)

Barh in un ostalerei vras ou deoé dichennet hag ino int e houlennas de zébrein ha d'ivein. (bis)

15 - Débet huei hag ivet er péh e rei vad doh kar itré trei soudard yevank en nouz e baséet! (bis)

Kar itré trei souclard yevank en nouz e baséet!.

Chetui arriù hantèr er goén. oeit er plah ha marùet (bis) Voici qu arrive le milieu du souper, la fille tomba morte.

Chetui arriù hantér er goén, oeit er plah ha marùet

20 Int 'lavar en eil d' egilé : - Men hi interam ni ? (bis)

Int 'lavar en eil d' egilé : - Men hi interam ni?

- Kasam-ti de jardrein hi zad idañ er fleur-de-lys. (bis)

Kasam-ti de jardrein hi zad idañ er feur-de-lys. $\mathrm{Ni}$ e bedo Dué aveitei man dei d'er barawis. (bis)

25 Deit e trei di goudi, hi zad yas d'er jardrein, Yoñ e glevas dré 'zañ en doar ur voéh melikantren (bis)

Yoñ e glevas dré 'zañ en doar ur voéh melikantren

- Me zad, me zad, mar me haret, men bé dein degont !(lisi)
Dans la grande ville de Paris lorsqu'ils furent arrivés, ils descendirent dans une grande hôtellerie

et là ils demandèrent à manger et à boire

- Mangez et buvez à votre contentement car vous passerez la nuit entre trois jeunes soldats!
Une jeune fille, fille d'un prince de Paris, se promenait dans le jardin de son père, sous la fleur de lys

les trois fils d'un jeune capitaine lui faisaient la cour.

et le plus jeune lui dit un jour :

Me zad, me zad, mar me haret, mem bé dein degoret!

30 Groeit en és mi trei dé er manì eit me inour goarnet! (bis) J'ai fait trois jours la morte pour mon honneur garder! 
Comme on peut le voir, la ligne mélodique est très simplifiée par rapport à son modèle français. À une formule ab/cd/be a succédé un nouveau schéma mélodique $\mathrm{dd} / \mathrm{be} / \mathrm{be}$ dont tous les éléments proviennent des deux dernières phrases de la mélodie française, mais agencés différemment.

Dans ces versions vannetaises, le texte qui est une traduction assez littérale de la version française commune, fait de la belle une princesse habitant Paris, Versailles ou Chateaubriant, et courtisée par trois jeunes capitaines (trois fils d'un jeune capitaine, trois jeunes officiers, trois gentilshommes). Dans une version de Belz où l'hôtesse, inquiète de savoir si la jeune fille est là de son plein gré, l'interroge, elle répond qu'elle a été enlevée dans la maison de ses parents. Dans une autre (Plouhinec), elle demande à son père qui la recherche dans son jardin de s'éloigner :

- Éloignez-vous, éloignez-vous, mon père, ne venez pas sur ma tombe !

Je suis ici depuis trois jours, depuis trois jours enterrée

pour garder mon honneur et je l'ai bien gardé.

\section{B. Les versions trégorroises}

On en connaît actuellement quatre, notées entre 1850 et $1980:$ les deux plus anciennes près de Lannion par l'un des tout premiers collecteurs de littérature orale, J. -M. de Penguern (1807-1856) ; une autre, un siècle plus tard, en 1952, à Ploubezre par l'abbé Louis Le Floc'h, plus connu sous son nom de plume Maodez Glanndour (texte et notation musicale) ; la dernière en 1980 à Trébeurden par Daniel Giraudon ${ }^{8}$.

Bien que directement adaptées des versions françaises comme les précédentes, elles s'en distinguent par une plus grande inventivité, tant sur le plan mélodique que sur celui du texte. On en jugera d'après la version de Ploubezre.

«Ar plac'h degaset d'an arme » (La fille amenée à l'armée)

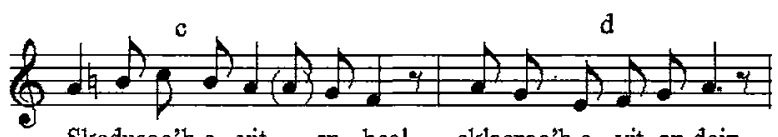

Skedusoc'b e-vit an heol, sklaeroc'h e - vit an deiz

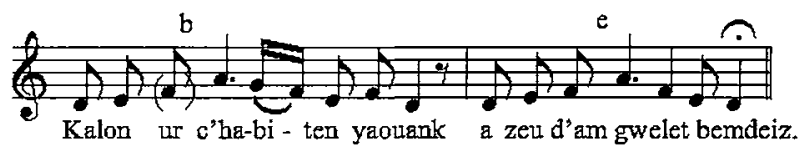

8. Cf. D. Giraudon, «Gwerz Katerinig», Brud Nevez, n69, 1983, pp.19-21. Texte breton et notation musicale. 
«Skedusoc'h evit an heol », collection L. Ar Floc'h [Louis Le Floc'h], Soaz An Digerc'her, Ploubêr, 1952.

1 Skedusoc'h evit an heol, sklacroc'h evit an deiz Kalon ur c'luabiten yaouank a zeu d'am gwelet bemdeiz.

- Deut-hu ganin, ma mestrez, ma mestrez deut ganin. Me ho kaso d'an anne, hoc'h eureujo goude-se

5 Me 'breno deoc'h un abid a vo pasimantet, Un tok kastol war ho penn, chwi heñvel ouzh ur gadred.

- Salokras, kahiten yajouank, d'an ame nan in ket Ma mamm 'zo war he gwele klañy, presiñ he maro 've graet.

Ma mamm 'zo war he gwele klañv, ar maro 'n he c'halon... 10 Ar placth yaouank ya d'an arme goll he reputasion.

- Montez, montez la belle. toilà mon cheual gris.

'Benn ma vo hantemoz sonet ni vo paset Pariz.

E-barzh ar barrouz C'hlaz, pa int bet arnuet,

En osteliri antreet, war an oaled azezet,

15 An ostiz, an ostizez, 'vel -henn dei o deus laret :

- Debonjour deoc'l-hu, plac'h yaouank, plac'hig diwar ar maez, Mar'd omp-ni d'ho fantazi, clisomel ganimp da vatezh.

Salokras, emei, ostizez, diwar ar maez nion ket, Me 'zo ganet a zenjentil, me 'zo bugel a di mat.

20 A zo deut er bloaz-mañ d'an ame, ya, dre ma bolontez vat.

- Ma vjjec'h ganet a zenjentil, bugel 'vel ma leret C'hwi vije duze 'barzh ar sal och evañ gwin klered.

Chwi vije duze barzh ar sal gant ho tud o koanian, Hag arc'hant en ho kodelloù ha c'hwi kontant da baeañ.

25 War ar propozioù-se, ar c'habiten yaouank anureas : - Koaniet, koaniet, Katarinig, kemeret ho plijadur, Hag ouzh kostez ur c'habiten, c'hwi a gousko a dra sur.

Sonet, sonet, trompeteu, ho piolañsoù dousamant, P'emañ ganimp o koanjañ fleurenn ar merched koant.

30 Oa ket hanter ar banked ar verc'h yaouank a zo marvet : - Seset, seset, trompeteu, ho piolañsoù dotsamant. Pa eo maro ma mestrezig, ma chalon 'zo dolant

Pelec'h vo interet Katarinig ma mestrez...? Nan eo barzh e jardin he zad dindan ur bod-lore 35 Me a bedo viti Doue, koulz en noz hag en deiz.

Nan eo barzh e jardin he zad dindan ur boked-roz Me a bedo viti Doue, 'vit mar gay d'ar baradoz.

- Digorit din ho tor, ma zad muiañ karet.

Tri deiz, ma maro am eus graet, ma enor' $m$ eus saveteet.

40 - Laret din, Katarinig, piw en deus hoc'h aleuvet Nan eo ur chabiten yaouank ha mab un den redouted 42 A oa o sevel an truajoì diwar ar baisanted..
Plus brillante que le soleil, plus claire que le jour, le cour diun jeune capitaine vient me voir chaque jour.

- Venez avec moi, ma maitresse, ma maîtresse, venez avec noi. je vous amènerai à l'amée et vous épouserai ensuite.

Je vous achèterai un habit passementé, sur vorre tère un chapeau de castor; vous ressemblerez à un ubleau. - Pardon, jeune capitaine, je n'irai pas à larmée : ma mère est dans son lit malade, on prépare sa mort. Maı mìre est dans son lit malade, la mort en son coeur... Une jeune fille qui va à larmée perd sa réputation.

lorsqu îl sera minuit sonné, nous aurons passé Paris. Dans la paroisse de Graces, lorsqu'ils furent arrivés, entrés dans l'hôtellerie, assis sur le foyer, l'hôtelier et l'hôrelière lui ont dit comme cela :

- Bonjour à vous, jeune fille, jeune fille de la campagne, si nous sommes à votre gré, restez chez nous comme servante.

- Pardon. dit-elle, hôtelière, je ne suis pas de la campagne, mon père est gentilhomme, je suis enfant de bonne maison. je suis venue cetre année à l'année de mon plein gré

- Si vous étiez fille d'un gentilhomme, enfant comme vous le dites, vous seriez là dans la salle à boire du vin clairet.

Vous seriez là dans la salle à souper avec vos gens, de l'argent plein les poches et contente de payer.

Sur ces propos, le jeune capitaine entra :

- Soupez, soupez, Catherinette, prenez votre plaisir, et au côté d'un capilaine. certainement vous coucherez.

Sonnez, sonnez, trompetres, et violons doucement, puiscjue soupe avec nous lat fleur des jolies filles.

On n'était pas au milieu du repas, la jeune fille est morte : - Cessez, cessez, trompettes, et violons doucement, puisqui est morte ma maitresse, mon coeur est dolent

Où sera enterré Catherinette ma maitresse... ? Si ce n'est au jardin de son père sous un laurier Je prierai Dieu pour elle, la nuit comme le jour.

Si ce riest au jarclin de son père sous un rosier Je prierai Dieu pour elle. qu elle aille au paradis..

- Ouvrez-moi la porte, mon père bien-aimé. Trois jours j'ai fait la morte, mon honneur jai sauvé.

Dites-moi, Catherinette, qui vous a enlevé sinon un jeune capinine, fils d'un homme redouté qui levait des taxes sur les paysans... 
a) La mélodie

Si l'on s'en tient à la notation qu'en donne son collecteur, elle ne comporterait que deux phrases au lieu des trois des versions françaises et vannetaises. Pourtant, un couplet sur trois (c. 7, 9, 12,14, 15 et 18) est bien un tercet. On peut donc supposer, bien que l'indication n'y figure pas, que l'une des deux phrases musicales était dite deux fois. C'est ce que laisse entendre le collecteur qui précise : « (la chanteuse) chantait sur un rythme assez irrégulier, abrégeant ou allongeant les phrases musicales selon les couplets. Ce n'était d'ailleurs pas facile de séparer ceux-ci les uns des autres car elle répétait deux fois la première moitié de l'air ».

Du point de vue de la structure mélodique, on remarque à nouveau que celle-ci n'utilise - mais cette fois en entier - que les deux dernières phrases mélodiques du tercet initial des versions françaises, soit la formule suivante $\mathrm{cd} / \mathrm{cd} / \mathrm{be}$ si, comme on peut le supposer, la première phrase était bien répétée deux fois.

\section{b) Le texte}

On notera plusieurs développements nouveaux.

Ainsi, aux deuxième et troisième couplets, la déclaration du jeune capitaine à la jeune fille est beaucoup plus circonstanciée : il lui promet de l'épouser si elle le suit à l'armée, et de lui acheter tout de suite un bel habit passementé. Mais elle refuse, prétextant que sa mère est mourante. De même le dialogue avec les hôteliers qui la prennent pour une fille de la campagne et lui proposent de l'engager comme servante... Elle se récrie alors et déclare qu'elle est fille de bonne maison : son père est gentilhomme et elle est venue à l'armée de son plein gré. Enfin, au dernier couplet, on remarquera la description qu'elle fait à son père de son ravisseur : le fils d'un homme redouté qui levait des taxes sur les paysans.

Par rapport à leurs sœurs vannetaises, les versions trégorroises semblent également plus soucieuses de ces précisions toponymiques ou anthroponymiques qui garantissent pour les auditeurs la véracité du récit. Trois d'entre elles donnent le prénom de la jeune fille - Katarinig (Catherinette) - et on vient de voir que la version de Ploubezre situait l'hôtellerie en Bretagne, "dans la paroisse de Grâces », contrairement d'ailleurs à l'ensemble des autres versions qui la localisent à Paris. Plus généralement, on voit que le ton est plus personnel, le discours plus précis, les détails plus abondants, comme pour rapprocher l'intrigue évoquée des situations psychologiques vécues par les chanteurs ou leurs auditeurs. 
Cette présentation rapide des versions bretonnes de « La Belle qui fait la morte », sous leurs deux aspects vannetais et trégorrois, nous a permis de suivre le processus d'accueil et d'adaptation d'une chanson française par une autre culture forte et vivante, ouverte au renouvellement mais soucieuse aussi de ne pas trop bouleverser le paysage poétique et mélodique que lui a légué la tradition. Parmi les thèmes qu'elle affectionne et qui l'habitent depuis toujours, celui de la mort et de l'autre monde est l'un des mieux représentés. On ne s'étonnera donc pas de le trouver présent, au moins par allusion, dans la plupart des poèmes chantés de l'ancienne tradition française qui ont fait carrière en Bretagne bretonnante : La Fille dans la tour, La Mariée chez Satan, La Dame au miroir d'argent, Le Flambeau d'amour... On trouverait certainement, dans la comparaison de chacune de ces chansons anciennes avec leurs versions bretonnes, matière à une étude riche d'enseignements ${ }^{9}$.

9. L'auteur tient à remercier, pour leur collaboration particulière à cet article, Robert Bouthillier, Christian Desbordes, Dany Guillou et Jean-Yves Monnat. 